\title{
Teléfonos móviles, redes sociales y praxis en adolescentes ${ }^{1}$
}

\section{Mobile phones, social networks and digital praxis in adolescents}

\author{
José Hernández-Ortega² \\ Universidad Complutense de Madrid, España \\ josehernandezortega@ucm.es \\ https://orcid.org/0000-0003-3556-5688 \\ LAURA Rayón-RUMAyOR \\ Universidad Complutense de Madrid, España \\ larayon@ucm.es \\ https://orcid.org/0000-0003-0339-8221
}

\section{Resumen:}

El binomio teléfonos móvil y RRSS en la adolescencia transciende lo tecnológico. Las prácticas de uso tan habituales en su vida cotidiana son ubicuas, pero relativamente invisibles para docentes y familias. Un conocimiento necesario para marcar una hoja de ruta en el diseño de propuestas educativas mediadas por tecnologías móviles. Mediante un estudio descriptivo longitudinal cuantitativo, se identifican niveles de uso y comportamiento con los teléfonos móviles en una muestra $(\mathrm{N}=645)$ escolar en ESO-Bachillerato, prestando especial atención a qué aplicaciones son las más empleadas, y el tiempo de uso en función del género y nivel educativo. Los resultados evidencian que el teléfono móvil no se percibe como un sistema de almacenamiento de información, se emplea con una finalidad comunicativa (RRSS) de las que se nutren las aplicaciones de edi-

\begin{abstract}
:
The association of mobile phones and social networks during adolescence surpasses technological considerations. These practices, while extremely common and ubiquitous in adolescents' daily lives, are relatively invisible to teachers and families. It is, therefore, necessary to establish a roadmap in the design of educational proposals enhanced by mobile technologies. Through a quantitative longitudinal descriptive study, levels of use and behavior with mobile phones are identified in a sample $(\mathrm{N}=645)$ of Secondary School and Baccalaureate students. Special attention, which we disaggregate by gender and education level, is paid to the most commonly used apps and to time of use. Results show that the mobile phone is not only identified as an information storage system, but it is also used for a communicative purpose (social media) which is
\end{abstract}

1 Como referenciar este artículo (How to reference this article):

Hernández-Ortega, J., \& Rayón-Rumayor, L. (2021). Teléfonos móviles, redes sociales y praxis en adolescentes. Educatio Siglo XXI, 39(3), 135-156. https://doi.org/10.6018/ educatio. 427011

2 Dirección para correspondencia (Correspondence address):

José Hernández-Ortega. Miembro del Grupo de Investigación DETECESE-UCM. Facultad de Educación. C./ Rector Royo Villanova, 1. 28840 Madrid (España) 
ción de imagen (fotografía-vídeo) y consumo de audiovisuales (vídeos-música). En el segundo año de ESO hay un incremento de aplicaciones y de tiempo empleado frente al móvil. Las diferencias de género suponen brechas significativas en cuanto al uso de RRSS, y el valor de la imagen un punto axiomático en ello. Una praxis que evidencia las amenazas que suscita su empleo, y el reto de diseñar una alfabetización digital ambiciosa que contemple usos responsables de las RRSS, conocimiento sumo informado de los contenidos digitales y la introducción de escenarios comunicativos multimodales para crear y compartir.

\section{Palabras clave:}

Teléfonos móviles; adolescencia; Redes Sociales; praxis digital. supported by image editing applications (photography-video) and audiovisual consumption (videos- music). In the second year of Secondary Education there is an increase in the use of apps and in the time spent using mobile phones. There are significant differences in relation to gender in the use of social media, being the value of the image an axiomatic point in this difference. Results show the threats inherent in the use of mobile phones and highlight the challenges when it comes to designing an ambitious praxis of digital literacy that contemplates responsible uses of social media, highly informed knowledge of digital content and the introduction of multimodal communication scenarios that favour sharing creative processes.

\section{Keywords:}

Smartphones; adolescents; social media; digital praxis.

\section{Résumé:}

L'association des téléphones portables et des médias sociaux à l'adolescence transcende la technologie. Les pratiques si courantes dans leur vie quotidienne sont omniprésentes, mais relativement invisibles pour les enseignants et les familles. Ces connaissances sont nécessaires pour établir une feuille de route pour la conception de propositions éducatives médiatisées par les technologies mobiles. Une étude descriptive longitudinale quantitative permet d'identifier les niveaux d'utilisation et de comportement avec les téléphones mobiles dans un échantillon $(\mathrm{N}=645)$ d'élèves du secondaire, en accordant une attention particulière aux applications les plus utilisées et au temps d'utilisation en fonction du sexe et du niveau d'éducation. Les résultats montrent que le téléphone mobile n'est pas perçu comme un système de stockage d'informations, il est utilisé à des fins de communication (RRSS), dont se nourrissent les applications d'édition d'images (photographie-vidéo) et de consommation audiovisuelle (vidéos-musique). Au cours de la deuxième année de l'OSE, on constate une augmentation du nombre d'applications et du temps passé sur les téléphones mobiles. Les différences entre les sexes représentent des écarts significatifs en termes d'utilisation du RRSS, et la valeur de l'image en est un point axiomatique. Une praxis qui met en évidence les menaces que représente leur utilisation, et le défi de concevoir une culture numérique ambitieuse qui envisage des utilisations responsables des médias sociaux, une connaissance hautement informée des contenus numériques et I'introduction de scénarios communicatifs multimodaux pour créer et partager.

\section{Mots clés:}

Téléphones mobiles; adolescence; réseaux sociaux; praxis numérique.

Fecha de recepción: 07-05-2020

Fecha de aceptación: 30-10-2020 


\section{Introducción}

España es un país hiperconectado a Internet. Los recientes estudios del INE (2019), Ditrendia (2019) y We are social (2020) arrojan el perfil de un país cuya práctica internauta sigue en constante crecimiento. El 90\% de la población está conectada a Internet, pero si se acota la franja de edad entre los 16 y 24 años (no trascienden datos oficiales en población más joven) la conectividad alcanza el 99.2\%. Datos que hacen necesario también un análisis del comportamiento de los más jóvenes por su percepción del mundo a través de una pantalla y su comportamiento heterogéneo ante sus iguales y con la generación de sus progenitores. Resulta relevante la presencia ubicua de Internet a través de dispositivos móviles, siendo España uno de los epicentros de la presencia social de teléfonos móviles con 54.4 millones de unidades, un 117\% sobre la población nacional. Este despliegue de dispositivos contribuye a que sea el método de acceso escogido entre los internautas: $96 \%$ para teléfonos móviles, $72 \%$ ordenadores y $41 \%$ Tablet (INE, 2019).

Una sociedad con un amplio y fomentado acceso dirigido hacia Internet, favorece en la población el uso y desarrollo de aplicaciones afines a tal propuesta. Y en esta tesitura, la población adolescente ocupa un lugar prioritario, son el máximo exponente de empleo y dedicación diaria frente a las Redes Sociales (RRSS). De los 318 minutos de consumo diario en Internet, 176 minutos ( 2 horas 54 minutos) se destinan a RRSS (INE). Su relevancia genera una demanda y uso que se incrementan exponencial y anualmente.

Las RRSS se perciben desde ópticas antagónicas. Por un lado, postulados como los de Herrera, Pacheco, Palomar y Zavala (2010), Tsitsika, Tzavela y Mavromatí (2013) o Fernández y Gutiérrez (2017) sostienen que las redes sociales generan distanciamiento y aislamiento social, reemplazando las relaciones humanas analógicas por las digitales como mecanismo de relación alternativo al establecido fuera de las redes. En contraposición a estos planteamientos, hallamos los sostenidos por investigaciones como la de Castells (2015), Campos (2008), y Reig (2012), quienes muestran que las RRSS generan efectos integradores del individuo en contextos de exclusión social y de consolidación de la identificación con la comunidad. La equidistancia mostrada por Morduchowicz (2012) y Winocur (2006) permite matizar que las RRSS no son las responsables directas en el cambio de conducta del individuo, especialmente 
del adolescente, sino la propia interacción del usuario con las distintas RRSS es la que genera la proyección de las relaciones personales analógicas en las digitales, con distintos sistemas de actitud y pretensiones de la autopercepción en una sociedad digitalizada y socializadora entorno a las mismas (Merino, 2011).

Necesariamente se ha de dibujar el proceso que ha llevado a este fenómeno tecnológico a consolidarse a escala nacional. El comportamiento humano en general y el adolescente en particular, ha virado de una interacción cara a cara entre iguales a una hiperrelación global cuyo escenario es la Red, incluso compartiendo espacio temporal y físico. El grado de modificación de las conductas sociales supone un punto de inflexión en las relaciones antropológicas contemporáneas en comparación con todas las generaciones previas, llegándose a la exclusión social o del grupo dominante si no se es miembro y partícipe activo de las distintas RRSS. Las conclusiones del informe Interactive Advertising Bureau Spain (IAB Spain, 2019) sobre el grado de penetración por número de usuarios de RRSS (periodo 2009-2019), evidencia que el crecimiento de usuarios adultos en nuestro país sufre una estancamiento en los últimos diez años. Entre la población que consolida la presencia de las redes destaca la población adolescente. Informes estatales como el INE (2019) arrojan datos en usuarios a partir de 14 años, aunque otros como IAB Spain (2019) ya ofrece muestras de población de 11 años que no hacen más que corroborar la necesidad de analizar los usos de la población adolescente desde su entrada en la etapa de Educación Secundaria Obligatoria (ESO) (Calvo y San Fabián, 2018).

El comportamiento que manifiestan los adolescentes en la ESO en la interacción con las RRSS (y por los dispositivos móviles) se rige por una autonomía no guiada por adultos, sino por la propia interacción, la tutela entre iguales o videotutoriales en Youtube. Gee y Hayes (2012) identifican patrones de comportamiento que justifican la intencionalidad de pertenencia a un grupo a través de las RRSS a través de una serie de características constitutivas: i) sus miembros son heterogéneos, desde expertos a noveles; ii) todos los usuarios son potenciales productores de contenidos, la creatividad y la originalidad son premisa para ellos; ii) el estatus y respeto se consiguen a través de distintos procesos y mecanismos; iii) el conocimiento, en múltiples focos, constituye una inteligencia colectiva desarrollada tanto individual como general y/o específica; y iv) el contenido se transforma merced a la interacción. 
Las investigaciones de Tejada et al. (2019), Fernández et al. (2015) y Lareki et al. (2017) concretan que la praxis de las RRSS protagonizada por los adolescentes arroja claroscuros. Siguiendo la tendencia general, el contenido más consumido y elaborado en la red sigue siendo el vídeo. En cuanto a la práctica diaria, sorprende que estos alumnos (llegando al $50 \%$ ) reconocen el intercambio de mensajes con perfiles que no conocen analógicamente; el 44\% afirma ser receptor de mensajes o imágenes desagradables por WhatsApp. Cuando se trata de su relación personal con las RRSS, el 13\% asevera haber sido objeto de alguna actuación molesta.

Un rescoldo de esperanza se atisba cuando estas cifras decrecen a medida que se los usuarios crecen y aumenta su experiencia y su capacidad de gestión ante las vicisitudes que padecen, tal y como evidencian los resultados de la investigación de Ballesta et al. (2014). Resulta interesante también el género en el análisis de uso de las RRSS. El estudio de Colás-Bravo et al. (2013), evidencia que no se hallan diferencias significativas entre sexos en cuanto a frecuencia de uso, pero sí en las motivaciones para su acceso, emocionales para los chicos y de tipo relacional para las chicas. A resultados similares Ilegan García et al. (2013), así como González \& Martínez (2018).

Las principales asociaciones atribuidas a los usuarios en edad de ESO y Bachillerato, es su grado de dependencia en virtud a múltiples factores. Rojas-Jara et al. (2019) han analizado factores asociados a la dependencia a las RRSS en relación al comportamiento, a la adicción a Internet, a trastornos de la personalidad, a la relación de adolescentes con éstas, a la relación de adolescentes con sus vínculos familiares, la calidad de vida y la conformación de la personalidad. La asociación de la adicción a las RRSS en adolescentes ya se tipifica como patología de comportamiento equiparable a las manifestadas por adicciones a estupefacientes (Carli et al., 2013; Müller et al., 2016; Vega \& Arancibia, 2019). La Comunidad de Madrid (2019) ha comenzado a intervenir en casos de adolescentes entre 12 y 17 años con una clara patología dependiente de la tecnología y las RRSS. Fruto de la investigaciones de la comunidad científica, se han establecido que algunos de estos trastornos se originan en aspectos derivados del uso nocivo de las RRSS provocando alteraciones como un claro narcisismo por agradar a la red de contactos (Casale et al., 2016), la obsesiva búsqueda por agradar en forma de likes o de reconocimiento del valor de lo publicado (Mazzoni et al., 2016), la humanización de 
los dispositivos tecnológicos (Menezes et al., 2019), la sensación de no gozar de lo que sucede en la red y constituye la moda a seguir (Przybylski et al., 2013), alteraciones en la rutina de sueño con el correlativo descenso del rendimiento escolar (Vernon et al., 2015), o insatisfacción con el propio cuerpo y con el momento vital en el que se encuentra el proceso evolutivo del adolescente (Wang et al., 2016). Circunstancias que inciden inevitablemente con un comportamiento disruptivo y tenso en el ambiente familiar, generando distanciamiento entre progenitores y adolescentes en el seno del conflicto familiar (Li et al., 2014). El momento evolutivo condiciona significativamente las relaciones analógicas frente a las digitales, puesto que a la brecha de conocimiento y uso de las redes entre padres e hijos, son estos últimos quienes desarrollan estrategias de gestión a partir de la propia experiencia o de la hallada en testimonios de su red de contactos digitales.

Los estudios de referencia evidencian que los dispositivos móviles, y en particular las RRSS, movilizan a los adolescentes a experimentar acciones e interacciones sociales de signo contrario, pero en cualquier caso con implicaciones antropológicas, sociales y psicológicas que no son ajenas a la Educación. Es imprescindible iniciar estudios que, a partir de los resultados de investigaciones sociales más amplias, permitan iniciar el necesario diálogo y conversación desde un mayor reconocimiento de los contextos escolares concretos en los que se genera un conocimiento irrenunciable para la Educación. La sociología digital, al igual que la antropología digital, reconocen la necesidad de iniciar debates interdisciplinares desde perspectivas catalizadoras que transiten desde estudios más generales a estudios locales, tal y como proponen desde la antropología Pink et. al (2016). Una mirada de la investigación que sitúa el análisis desde la teoría y desde lo que ésta permite pensar (Hernández-Hernández, 2019), y así contribuir a comprender el uso y acciones con lo digital, y sus implicaciones para, como en nuestro caso, articular e impulsar la formación de los adolescentes (Bañares-Mediavila \& Rayón-Rumayor, 2017). Un modo de concebir la investigación sin renunciar a un conocimiento específico de los contextos reales compartidos que habitan profesores, estudiantes y familias. Sin ese diálogo, la investigación en relación con lo digital perdería su sentido para transformar lo educativo. Ello tiene la virtualidad de servir a otros investigadores y profesionales, interesados en articular propuestas educativas que respondan a un marco social y cultural específico y concreto. 
Más si cabe, ante el protagonismo que la COVID-19, de un modo inesperado, ha otorgado a los dispositivos móviles para generar otros espacios y situaciones de aprendizajes hasta ahora poco transitadas en muchos centros educativos. La brecha digital intergeneracional se ha agudizado en beneficio de una generación, la adolescente, que trasciende la comunicación bimodal para desarrollarse como nativos multimodales en un ecosistema comunicativo multimodal en la que son prosumidores activos y consolidados (Scolari, 2013). La multimodalidad semiótica constituye uno de los elementos de identificación con el grupo y de cohesión social.

\section{Material y métodos}

\section{Objetivos}

Esta investigación tiene como principal objetivo obtener una comprensión bien informada y contextualizada de la praxis adolescente en el uso del teléfono móvil y RRSS en población en edad escolar en ESO y BachiIlerato en función del género y nivel educativo. Para ello, el primer objetivo específico se orienta a identificar niveles de uso y comportamiento con los teléfonos móviles atendiendo a qué actividades llevan a cabo, y el tiempo de uso empleado; el segundo objetivo pretende identificar qué RRSS sociales utilizan los adolescentes y la percepción del riesgo asociado a su uso.

\section{Método}

Se ha elaborado un estudio descriptivo longitudinal de investigación cuantitativa. Para Mateo (2012, p. 198) esta elección metodológica busca la realización de descripciones precisas y muy cuidadosas de fenómenos educativos, propias de los primeros estadios de investigación que proporcionan los datos necesarios para la elaboración de nuevos planteamientos teóricos o profundización en investigaciones posteriores, sin renunciar a contrastar el conocimiento generado con resultados de otras investigaciones. 


\section{Muestra}

La muestra se compone de 645 individuos, una muestra longitudinal de estudiantes de $1^{\circ}$ ESO a $2^{\circ}$ Bachillerato de un centro concertado de la periferia Madrid. La muestra atiende a parámetros de probabilidad e incidencia en función de la accesibilidad de los informantes (Mateo, 2012) con un total de 645 individuos, $(54.26 \%, \mathrm{~N}=350$ chicas y $45,74 \%, N=295$ chicos). Se confirma, según el estudio previo de Gialamas et al (2013), la ausencia de sesgo por género en estas etapas educativas.

Que la muestra se circunscriba a un centro escolar tiene un valor en sí mismo, generar un conocimiento como punto de referencia fundamental a la hora de la toma de decisiones en materia educativa. La elección del mismo se justifica por ser un centro de referencia en Tecnología Educativa en la Comunidad de Madrid, y por conveniencia, desde el primer momento se tuvo acceso garantizado a la casi totalidad de la población que ha conformado la muestra.

La muestra por nivel académico, para una población de 660 estudiantes, se articula así: $1^{\circ}$ ESO registra un total de 131 informantes $(20.31 \%)$, en $2^{\circ}$ y $3^{\circ}$ ESO con 130 cada uno (20.16\%), $4^{\circ}$ ESO con $102(15.81 \%)$, $1^{\circ}$ Bachillerato con 86 (13.33\%) y $2^{\circ}$ Bachillerato con 66 (10.23\%). El cambio significativo del número de informantes de la etapa ESO al Bachillerato, además de la obligatoriedad también viene condicionada por el tipo de educación (concertada para ESO y privada para Bachillerato). Esta disposición del alumnado también nos permite analizar el condicionante de la edad de los informantes en franjas. Entre los 11 y 12 años se encuentran 111 informantes (17.21\%), entre los 13 y I4 años 261 (40.47\%), entre los 15 y 16 años 176 (27.29\%) y entre los 17 y 18 años, $97(15.04 \%)$.

\section{Instrumento}

La recopilación de datos para responder a los objetivos del presente estudio es un cuestionario digital a través de Microsoft Forms ${ }^{3}$, diseñado específicamente para la investigación, completado en septiembre de 2019 y compuesto por 20 preguntas con la intención de mostrar los niveles de uso del teléfono móvil y de la interacción que mantienen con

3 Cuestionario disponible para su consulta en: https://bit.ly/395E13S 
las distintas aplicaciones móviles, en especial las RRSS. Esta escala se forma a partir de preguntas con una escala Likert (Nada, Poco, Bastante, Mucho), otras de elección múltiple en cuanto a redes sociales en las que se tiene perfil (figuras 3 y 4), más precisas de verificación en cuanto a identificación de términos concisos, como también a las relativas a franjas horarias (figura 4) que proporcionan un acotamiento de los datos, como evidencias tangibles y empíricas de los hábitos y prácticas de uso de móviles y RRSS de los estudiantes.

\section{Resultados}

Los resultados se exponen a continuación, organizados en epígrafes, y atendiendo a los objetivos específicos del estudio para facilitar su lectura. Se presentarán datos globales para el total de la muestra que den cuenta de la tendencia general de usos con el teléfono móvil, que serán posteriormente desglosados por nivel educativo y género para analizar la homogeneidad o no en función ambas dimensiones. Procederemos de igual modo para el caso particular del uso de RRSS.

\section{A) Usos del teléfono móvil y tiempo de exposición por nivel educativo y género}

En relación a cuántos estudiantes disponen de teléfono móvil propio, un $95.50 \%(\mathrm{~N}=616)$ responden afirmativamente frente al $4.50 \%(\mathrm{~N}=29)$ que responden negativamente. De este porcentaje, en cuanto a sistemas operativos de mayor uso, Android e iOS se erigen como únicas representaciones. El $75.57 \%(\mathrm{~N}=481)$ son usuarios de Android por el $22.02 \%$ $(\mathrm{N}=142)$ que lo son de iOS. En todos ellos, la cantidad de aplicaciones móviles (Apps) instalada se distribuye de forma heterogénea atendiendo a género y nivel educativo. Con menos de 10 Apps instaladas, el grupo predominante es el de $2^{\circ}$ Bachillerato, en el caso de las chicas con el $45.24 \%(\mathrm{~N}=19)$, y el de $1^{\circ} \mathrm{ESO}$ en el de los chicos con $34 \%(\mathrm{~N}=17)$ y las chicas con un $33.33 \%(\mathrm{~N}=27)$.

Entre 11 y 20 Apps destacan $2^{\circ}$ ESO las chicas con $47.83 \%(\mathrm{~N}=33)$, $3^{\circ} \mathrm{ESO}$ un $47.62 \%(\mathrm{~N}=30)$; y en $1^{\circ}$ Bachillerato con $48.89 \%(\mathrm{~N}=22)$ y $2^{\circ}$ Bachillerato con $45.83 \%(\mathrm{~N}=11)$ entre los chicos. El grupo de 21 a 30 Apps, destaca $2^{\circ} \mathrm{ESO}$ con $28.99 \%(\mathrm{~N}=20)$ por las chicas y $4^{\circ} \mathrm{ESO}$ con 
$31.25 \%(\mathrm{~N}=15)$ por los chicos, son los grupos relevantes. En el último sector, el de más de 30 Apps instaladas, es $2^{\circ}$ Bachillerato con $19.05 \%$ $(\mathrm{N}=8)$ entre las chicas y $2^{\circ} \mathrm{ESO}$ con $36.07 \%(\mathrm{~N}=22)$ entre los chicos, quienes atesoran mayor cantidad de Apps instaladas, como se evidencia en la Figura 1:

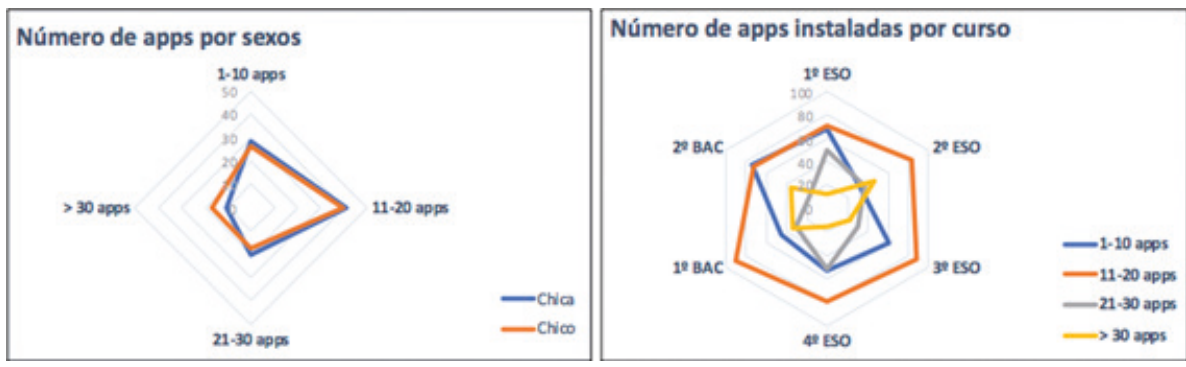

Figura 1. Número de apps instaladas atendiendo a sexos y nivel educativo de los informantes

En relación al uso que se dispensa a una serie de actividades propuestas a través del móvil, los resultados significativos son los que engloban a las respuestas Mucho/Bastante, mucho más frecuentes que las de Poco/Nada. Los resultados arrojados en la figura 2, corroboran que la actividad con mayor uso es escuchar música $(88.22 \%, \mathrm{~N}=569)$, siendo las chicas $(93.43 \%, \mathrm{~N}=327)$ quienes protagonizan un uso destacado frente a los chicos $(82.03 \%, \mathrm{~N}=242)$, una distribución homogénea entre ellas y heterogénea entre ellos, como puede apreciarse en la diferenciación entre el primer ciclo de ESO y el segundo ciclo y Bachillerato.

En cuanto a actividades concretas, destaca WhatsApp con un uso general del $80.78 \%(\mathrm{~N}=521)$ con valores heterogéneos por género $(84.29 \%$, $\mathrm{N}=295$ para las chicas y $76.61 \%, \mathrm{~N}=226$ para los chicos) y por nivel académico, con valores progresivos proporcionales al curso en el que se hallan los informantes. Datos semejantes, aunque inferiores, a los registrados en los valores de uso en otras RRSS con la misma heterogeneidad, como veremos en el siguiente apartado. En cuanto a actividades diferenciadas, ver vídeos es una actividad con resultados muy heterogéneos a favor de los chicos $(81.36 \%, \mathrm{~N}=240)$ frente al uso menos activo de las chicas $(62.29 \%, \mathrm{~N}=218)$. En cambio, las actividades en las que se invierte el predominio son las de hacer fotos, con mayor protagonismo de las chicas $(65.71 \%, \mathrm{~N}=230)$ frente al uso de los chicos $(19.32 \%, \mathrm{~N}=57)$, así 
como en el visionado de series, donde ellas $(41.43 \%, \mathrm{~N}=145)$ muestran más actividad que ellos $(26.78 \%, \mathrm{~N}=79)$.

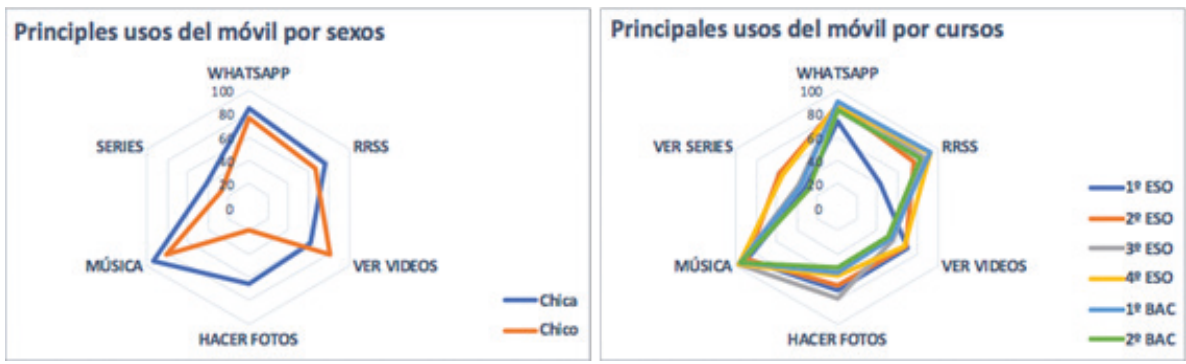

Figura 2. Principales usos del móvil atendiendo a sexos y nivel educativo de los informantes.

Los resultados procedentes que suscitan el valor de la investigación aquí presentada en relación con el tiempo de uso, arrojan el siguiente análisis. En primer lugar, el tiempo de uso que emplean con sus dispositivos móviles, como se refleja en la figura 3, evidencia cómo el género y nivel educativo arrojan datos diferenciados en el aumento de horas frente a la pantalla del móvil. A mayor tiempo, las alumnas $(27.44 \%, \mathrm{~N}=177)$ casi duplican su presencia en relación a los alumnos $(16.28 \%, \mathrm{~N}=105)$ en más de dos horas diarias. Proceso inverso se manifiesta en los informantes más jóvenes, quienes son los que manifiestan mayor restricción de uso en todas las franjas propuestas. En el caso de la diferenciación por nivel académico, esta heterogeneidad $2^{\circ}$ Bachillerato $(71.21 \%, \mathrm{~N}=47)$ arroja datos muy superiores a los de $1^{\circ} \mathrm{ESO}(19.85 \%, \mathrm{~N}=26)$.
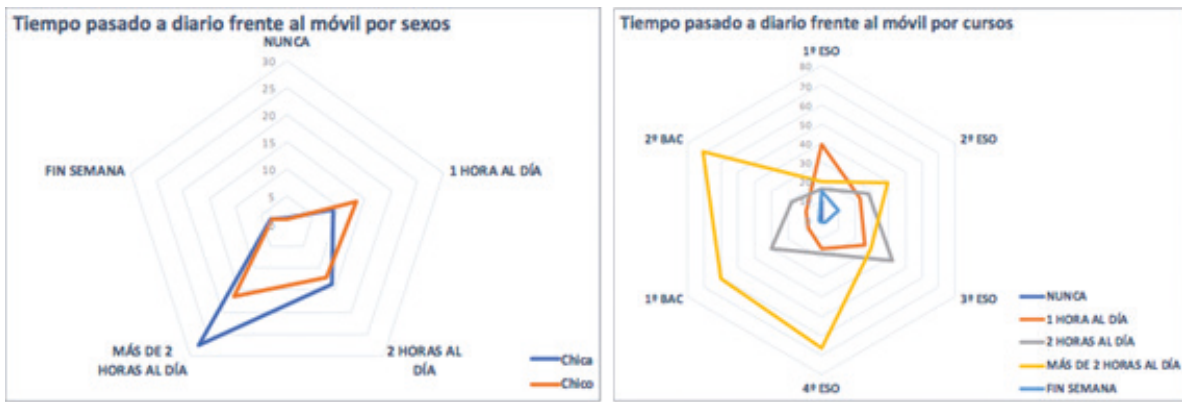

Figura 3. Tiempo pasado a diario frente al móvil atendiendo a sexos y nivel educativo de los informantes. 


\section{B) Usos de las RRSS y percepción del riesgo por nivel educativo y género}

En relación a qué alumnos poseían perfiles en alguna de las RRSS más prolíficas entre el alumnado, se constata el uso extendido de estas, en especial las relativas a contenido multimodal (Figura 4). Las más demandadas YouTube (81.55\%, N=525) e Instagram (77.21\%, N=498). Se erigen como las dos redes predominantes, frente a Ask.me $(4.96 \%, \mathrm{~N}=32)$ de menor presencia general. Un segundo bloque de redes lo constituyen aquellas que registran usuarios en más de la mitad de los encuestados: Sptify (58.44\%) y Snapchat (53.79\%).

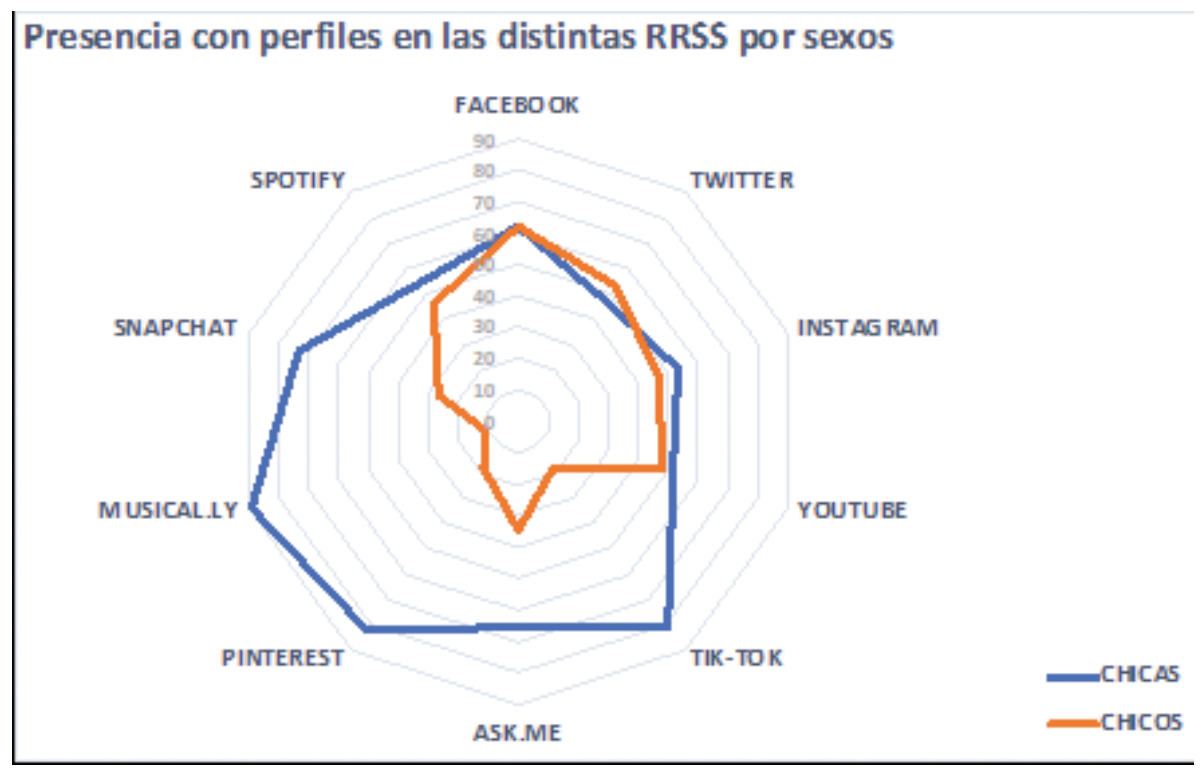

Figura 4. Análisis de la presencia en RRSS por sexo de los informantes.

La distribución por género (figura 5) se concreta en que los chicos prefieren Youtube $(86.78 \%, \mathrm{~N}=256)$, Twitter $(29.15 \%, \mathrm{~N}=86)$ y Facebook $(20.68 \%, \mathrm{~N}=61)$. Por parte de las chicas, las redes preferidas son Snapchat (72.86\%, N=255), Tik-tok (48\%, N=168), Musical.ly (43.71\%, $\mathrm{N}=153)$ y Pinterest (33.43\%, $\mathrm{N}=117)$. Sólo en el caso de Spotify se verifica un uso homogéneo en cuanto a género: $58.98 \%(\mathrm{~N}=174)$ para los chicos y el $58.00 \%(\mathrm{~N}=203)$ de las chicas. Una tercera perspectiva es la ofrecida por la preferencia de las distintas RRSS atendiendo al nivel educativo, donde se diferencian redes de mayor preferencia por informantes de $4^{\circ}$ ESO y Bachillerato, como Facebook, Twitter, Instagram, Youtube y 
Spotify. Los informantes más jóvenes son aquellos que tienen en Tik-Tok y Musical.ly sus redes preferentes. Se identifica una homogeneidad en cuanto a valores en el uso de Pinterest como red de equilibrio entre los distintos niveles educativos, como así se corrobora en la Figura 5.

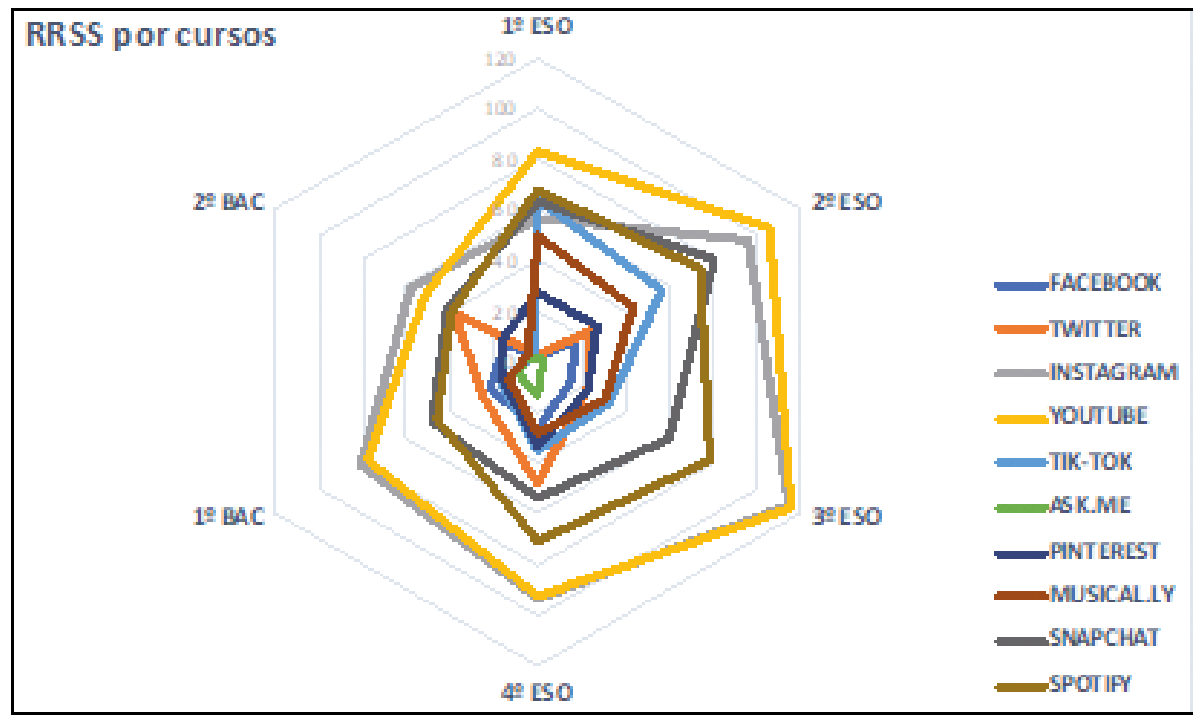

Figura 5. Preferencia de uso de las distintas RRSS por nivel educativo.

La combinación de empleo de WhatsApp, hábitos de uso y RRSS abre la puerta a seis factores de análisis en cuanto a riesgos. A la pregunta de si identifican qué es y/o en qué consisten los principales riesgos derivados de su uso, las respuestas ofrecen resultados claros en cuanto a identificación. Cyberbulling $(97.98 \%, \mathrm{~N}=632)$ y Violencia de género $(98.60 \%, \mathrm{~N}=636)$ son identificados por la práctica totalidad, frente a la sextorsión (51.47\%, N=332), Malware (37.67\%, N=243), Grooming $(30.54 \%, \mathrm{~N}=197)$ y Pishing $(25.12 \%, \mathrm{~N}=162)$ conocidos por un número muy inferior de informantes. De éstos, destaca que se produce una clara homogeneidad de resultados en cuanto a género pero no en cuanto a nivel educativo, donde los resultados son heterogéneos a favor de los informantes de los tres últimos cursos escogidos, quienes aglutinan los resultados más altos en comparación con los informantes de los tres cursos iniciales de ESO, como se puede comprobar en la figura 6. 


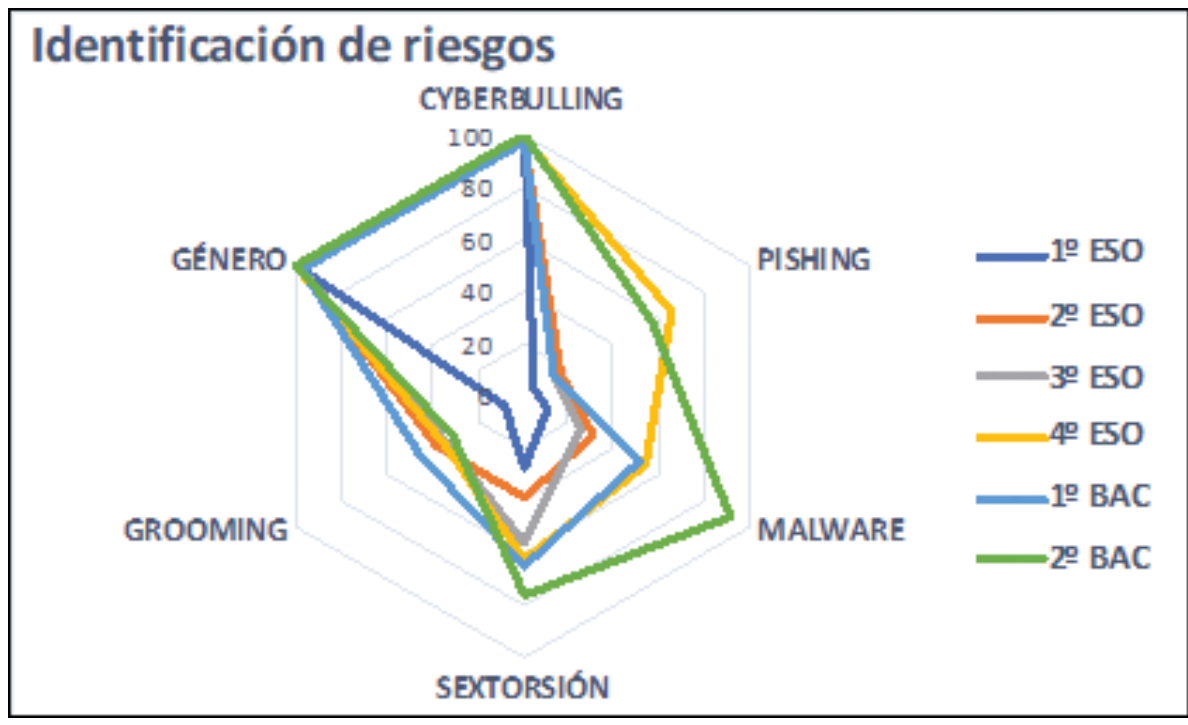

Figura 6. Identificación de los riesgos de las distintas RRSS por nivel educativo.

\section{Discusión y conclusiones}

El presente artículo ha pretendido mostrar distintos aspectos del uso de los dispositivos móviles en un contexto escolar determinado. En relación con el primer objetivo específico, el teléfono móvil no se percibe como un sistema de almacenamiento de información sin criterio, se emplea como un mecanismo donde se instalan un número reducido de aplicaciones con finalidad comunicativa (RRSS) de las que se nutren las aplicaciones de edición de imagen (fotografía y vídeo) y las audiovisuales (visualización de vídeos y escucha de música) como se puede comprobar en la figura 3. Los adolescentes son activos prosumidores de contenido multimodal, pero tambien son productores de mensajes conformados por más de un sistema de representación. Ello debiera hacernos reflexionar sobre la continuidad o discontinuidad entre las formas de leer y escribir que los adolescentes despliegan en los contextos formales y las desplegadas en los contextos informales soportadas en el teléfono móvil. El consumo de contenido audiovisual a través de las aplicaciones de edición de fotografía y vídeo evidencian que los adolescentes transcienden el canon de la lectura y escritura gutenberiana de la escuela. Sería interesante poner a su disposición experiencias educativas mediadas 
por los dispostivos móviles que abarquen un concepto de alfabetizacion digital ambicioso.

Por un lado, si los adolescentes son usuarios de entornos multimodales, consumidores de series y otros contenidos audiovisuales, como se puede comprobar en la figura 2, sería pertinente contemplar una formación que les faculte para comprender la lógica de la distribución de las plataformas transmedia mediante las que se distribuye lo audiovisual. La ubicuidad del teléfono móvil conlleva prácticas basadas en la inmediatez, y delegar, casi de un modo inconsciente, en un algoritmo la libertad de elección para consumir contenidos. La hibridación de funciones del teléfono móvil conlleva entornos discursivos complejos que combinan elementos de la narrativa audiovisual clásica con nuevos lenguajes, de modo que esas prácticas son invisibles para los usuarios. Parece necesario que los adolescentes comprendan quién comunica y por qué, e identifiquen las lógicas comerciales que sostienen las aplicaciones que utilizan los adolescentes. Estos tienen que aprender a detectar cuándo son blanco de avisos publicitarios, y cómo la información que suministran puede ser utilizadas por determinadas corporaciones. Por otro lado, sería interesante poner en situación a los adolescentes en producir mensajes multimodales, pero integrados en propuestas que puedan combinar el canon lector gutenberiano con la nueva cultura "read \& write", ligada a la interactividad e hibridación de los sistemas de representación soportados en las aplicaciones que utilizan en sus dispositivos móviles (Bañares \& Rayón, 2017).

Estas reflexiones son importantes, más si cabe, porque como los datos evidencian es en el segundo año de ESO cuando se produce el incremento de aplicaciones y de tiempo empleado frente al móvil. Si contrastamos estos resultados con las aplicaciones en Bachillerato, estas prácticas siguen una tendencia a nivel europeo (Tejada et al., 2019). Téngase en cuenta que el aumento significativo del tiempo destinado al teléfono móvil genera una dependencia y falta de control por el momento evolutivo en el que se produce, la socialización digital no sigue los patrones de la analógica porque en la etapa educativa en la que los adolescentes se encuentran, el proceso analógico todavía no se ha consolidado con las herramientas y estrategias necesarias para una madurez en la gestión y la toma de decisiones (García y Heredia, 2017; Fernández y Gutiérrez, 2017; Malo-Cerrato, Martín-Perpiñá y Viñas-Poch, 2018).

Los resultados por género si cruzamos los resultados en relación con 
el tiempo de uso y aplicaciones disponibles en el teléfono móvil arroja una análisis interesante. Como se pudo comprobar en la figura 3, el aumento de horas frente a la pantalla del móvil, tanto para chicos y chicas, se incrementa en $1^{a}$ y $2^{a}$ de Bachillerato. Pero el número de aplicaciones instaladas para el grupo de las chicas se reduce de un modo inversalmente promocional al nivel educativo, como se evidencia en la figura 1. Los chicos siguen la tendencia contraria, el número de aplicaciones instaladas se va incrementado. Estos resultados evidenciarían que las adolescentes hacen un uso más limitado de aplicaciones que sus pares al Ilegar a $2^{\text {a }}$ de Bachillerato. De estos resultados se puede colegir que los chicos a mayor nivel educativo son más permeables y proclives a utilizar más aplicaciones nuevas que las chicas. Lo que podría estar indicando cierta desigualdad en el desarrollo de saberes y competencias para desenvolverse y aprovechar la riqueza de lo digital.

En cuanto a los usos del teléfono móvil más populares, la muestra de resultados mantiene la línea marcada por las investigaciones previas de Tejada et al. (2019), Fernández et al. (2015), y Lareki et al. (2017), el uso de las RRSS es la praxis más extendida entre los adolescentes, siendo unas RRSS más populares que otras. Los datos confirman que WhatsApp se erige como la red social más empleada por los adolescentes seguida, al mismo nivel por Youtube, corroborando también las tendencias indicadas en los últimos años en los distintos estudios (IAB Spain, 2019; INE, 2019; We are social, 2020; Ditrendia, 2019) que confirman el auge de Instagram y el declive de Facebook y Twitter entre los adolescentes. Esto es atribuible a la tendencia de uso en los medios, así como a la mercadotecnia de las principales marcas comerciales, quienes fomentan determinadas redes en detrimento de otras. De nuevo, aparece el componente de seguridad al que nos referíamos previamente, la necesidad de trabajar en la escuela una alfabetización digital que transcienda lo instrumental, y permita a los adolescentes la toma de conciencia sobre el papel global de la publicidad, promoción y patrocinio en el funcionamiento de las RRSS.

En relación al segundo objetivo específico de la investigación, los usos de las RRSS y la percepción del riego, los resultados evidencian que las RRSS se erigen como una de las principales fuentes de realización personal de los informantes, a las que destinan una importante cantidad de tiempo que se incrementa conforme avanzan en su trayectoria académica. De su interacción se pueden confirmar diversos comportamientos 
y usos derivados de los resultados de la investigación. En cuanto a jerarquía entre las distintas redes sociales, WhatsApp es la que se emplea durante más tiempo y la que más usuarios registra de cuantas se utilizan por los informantes, desbancando a Facebook y Twitter como referencia de las RRSS en beneficio de Yotube, Instagram y Spotify (Ditrendia, 2019; García y Nazaret, 2017; IAB Spain, 2019; Malo-Cerrato et al., 2018). Un segundo aspecto a destacar (figura 3) es el del uso de la imagen (hacer fotos y vídeos) que se entiende como independiente de la utilización de estos mismos usos si se hace desde una red social audiovisual, especialmente Instagram y YouTube.

Las diferencias de género suponen brechas significativas en cuanto al uso en la linea del trabajo previo de Colás et al., (2013). Los chicos escogen prioritariamente Facebook, Twitter y Youtube, mientras que ellas escogen redes con alta presencia de la imagen y el vídeo: Tik tok, Ask. me, Pinterest, Musical.ly y Snapchat. Datos interpretables desde los modelos de referencia de ellos (deportistas) e influencers (ellas) quienes definen un uso prioritario hacia unas u otras redes. Asimismo, el valor de la imagen supone un punto axiomático en su uso, puesto que es la necesidad de autoafirmación y configuración de la propia personalidad (Rubio-Romero, Jiménez y Barón-Dulce, 2019) como la de proyectar una imagen a las redes donde se genera dependencia narcisista por la necesidad de sentirse realizado (Casale et al., 2016). Pero el valor de la imagen evidencia también el potencial que tienen las RRSS para que los adolescentes desplieguen nuevas formas de lectura y escritura que integran imagen, pero también sonido, emoticonos y texto escrito. Nuevas formas de comunicarse e interaccionar con los otros en las que indudablemente despliegan procesos semióticos multimodales, y por ello, la construcción de mensajes a partir del despliegue de significados complejos y creativos al utilizar una combinación de distintos sistemas de representación. Relacionarse a través de las RRSS conlleva que los adolescentes se conviertan en productores de textos para ser vistos, leídos y escuchados en un contexto que integra o excluye del grupo en función de la presencia e interacción digital. De nuevo, aparece la necesidad de repensar qué saberes y competencias despliegan cuando utilizan las RRSS, y cuáles despliegan en los contextos educativos formales. Una tarea ineludible para llenar de contenido una alfabetización digital coherente con estas nuevas formas de comunicación e interacción social.

Pero esta praxis genera también la obligación de conocer las prin- 
cipales amenazas que suscita el uso de las RRSS. Los datos obtenidos revelan que cyberbulling y violencia de género se identifican claramente, pero no así otras como el pishing, malware, sextorsión y grooming. Las campañas de concienciación surgen efecto en los primeros, pero sólo se perciben resultados adecuados en aquellos niveles en los que la formación específica curricular en TIC ( $4^{\circ}$ ESO y Bachillerato) contribuye a consolidar las competencias digitales necesarias para su correcta gestión. Resultados similares a los obtenidos por Fernández et. al, (2015), los adolescentes necesitan profundizar en los principios de ética y responsabilidad social ante el derecho a la intimidad de sus iguales. Al igual, que tomar conciencia que el uso de las RRSS conlleva un plus de vigilancia y atención por parte del usuario para no ser objeto de estafas, o verse inmersos en prácticas inmorales y deleznables basadas en el diseño de perfiles falsos en estos entornos de interacción social.

La praxis adolescente de uso del teléfono móvil y RRSS evidencia una oportunidad para la formación de los adolescentes. Aprovechar el potencial formativo del teléfono móvil implicaría dotar de un sentido pedagógico a los comportamientos y actividades que los adolescentes llevan a cabo, en la línea que los resultados obtenidos indican. Desde los centros educativos es necesario debatir y analizar los contenidos de la alfabetización digital para integrar estos dispositivos móviles en propuestas de trabajo ambiciosas y transversales. El consumo de contenido audiovisual desde el conocimiento de las lógicas de marketing y big-data, la construcción de textos multimodales en proyectos colobarativos para ser compartidos y comunicados desde una lógica transmedia, así como el conocimiento claro y preciso de los riesgos asociados al uso de las RRSS, parecen contenidos imprescindibles. Unos planteamientos que rompen con la prohibición del uso de los dispositivos móviles en los centros escolares, y que con carácter general las administraciones educativas de algunas Comunidades Autónomas del Estado español ha normativizado.

Finalmente, se considera necesario abrir una vía de investigación exploratoria en el pensamiento crítico hacia comportamientos en las distintas RRSS como también la pragmática del discurso multimodal que protagonizan los alumnos, con especial énfasis en los cambios generacionales que se suscitan de la innovación tecnológica, la pragmática del discurso y la semiótica comunicativa que se persigue en la interacción entre adolescentes y redes sociales. 


\section{Referencias}

Ballesta, J., Lozano, J., Cerezo, M. C., \& Soriano E. (2014). Internet, redes sociales y adolescencia: un estudio en centros de educación secundaria de la región de Murcia. Revista Fuentes, 16, 109-130. http://dx.doi.org/10.12795/revistafuentes.2015.i16.05

Bañares-Marivela, E., \& Rayón-Rumayor, L. (2017). Multimodal Narratives and iPad in Second Language Teaching. In D. Tafazoli, \& M. Romero (Ed.), Multiculturalism and Technology-Enhanced Language Learning (pp. 57-79). IGI Global. http:// doi:10.4018/978-1-5225-1882-2.ch004

Campos Freire, F. (2008). Las redes sociales trastocan los modelos de los medios de comunicación tradicionales. Revista Latina de Comunicación Social, 63, 287-293. https://doi.org/10.4185/RLCS-63-2008-767-287-293

Calvo, S., \& San Fabián, J.L. (2018). Redes Sociales y Socialización Afectiva de las Personas Jóvenes: Necesidades Docentes en Educación Secundaria Obligatoria. Revista Iberoamericana sobre Calidad, Eficacia y Cambio en Educación, 16(2)mar. https:// dx.doi.org/10.15366/reice2018.16.2.001.

Carli, V., Durkee, T., Wasserman, D., Hadlaczky, G., Despalins, R., Kramarz, E., Wasserman, C., Sarchiapone, M., Hoven, C. W., Brunner, R., \& Kaess, M. (2013). The Association between Pathological Internet Use and Comorbid Psychopathology: A Systematic Review. Psychopathology, 46, 1-13. https://doi.org/10.1159/000337971

Casale, S., Fioravanti, G., \& Rugai, L. (2016). Grandiose and Vulnerable Narcissists: Who Is at Higher Risk for Social Networking Addiction?. Cyberpsychology, behavior and social networking. 19. 510-515. https://doi.org/10.1089/cyber.2016.0189

Castells, M. (2015). Networks of outrage and hope. Social movements in the internet age. Polity Press.

Colás-Bravo, P., González, T., \& de Pablos, J. (2013). Young People and Social Networks: Motivations and Preferred Uses. [Juventud y redes sociales: Motivaciones y usos preferentes]. Comunicar, 40, 15-23. https://doi.org/10.3916/C40-2013-02-01

Comunidad de Madrid (2019). Servicio de atención en adicciones tecnológicas. https:// tinyurl.com/wn3qlyc

Ditrendia (2019): Informe Ditrendia: Mobile en España y en el Mundo. Madrid: Ditrendia Digital Marketing Trends. https://tinyurl.com/tvhstc4

Fernández Rodríguez, E., \& Gutiérrez Pequeño, J. M. (2017). La socialización de los jóvenes interconectados: Experimentando la identidad en la sociedad aumentada. Profesorado. Revista de Currículum y Formación de Profesorado, 21(2), 171-190. https:// tinyurl.com/vns83l4

Fernández, J., Peñalba, M. A., \& Irazabal, I. (2015). Hábitos de uso y conductas de riesgo en Internet en la preadolescencia. [Hábitos de uso y conductas de riesgo en Internet en la preadolescencia] Comunicar, 44, 113-120. https://doi.org/10.3916/C44-201512

García, E., \& Heredia, N. (2017). Emociones y redes sociales en adolescentes, Revista de estudios e investigación en Psicología y Educación, 13. https://doi.org/10.17979/ reipe.2017.0.13.2131

García-Jiménez, A., López de Ayala, M.C., \& Catalina, B. (2013). The Influence of Social 
Hernández Ortega, J. y Rayón-Rumayor, L. (2021). Teléfonos móviles, redes sociales y praxis en adolescentes. Educatio Siglo XXI, 39(3), 135-156.

Networks on the Adolescents' Online Practices. [Hábitos de uso en Internet y en las redes sociales de los adolescentes españoles]. Comunicar, 41, 195-204. https://doi. org/10.3916/C41-2013-19

Gee, J., \& Hayes, E. (2012). Nurturing Affinity Spaces and Game-based Learning. En C. Steinkuehler, K. Squire, \& S. Barab (Eds.), Games, Learning, and Society: Learning and Meaning in the Digital Age (pp. 129-153). Cambridge: Cambridge University Press.

Gialamas, V., Nikolopouiou, K., \& Kutromanos, G. (2013). Student teachers' perceptions about the im pact of Internet usage on their learning and Jobs. Computers \& Education, 62, 1-7. https://doi.org/10.1016/j.compedu.2012.10.012

González, E., \& Martínez, N. (2018). Redes sociales como factor incidente en el área social, personal y académica de alumnos de Educación Secundaria Obligatoria. Tendencias Pedagógicas, 32, 133-146. http://dx.doi.org/10.15366/tp2018.32.010

Hernández-Hernández, F. (2019). La perspectiva post-cualitativa y la posibilidad de pensar en 'otra' investigación educativa. Educatio Siglo XXI, 37(2), 11-20. https://doi. org/10.6018/educatio.386981

Herrera Harfuch, M. F., Pacheco Murguía, M. P., Palomar Lever, J., \& Zavala Andrade, D. (2010). La acción a Facebook relacionada con la baja autoestima, la depresión y la falta de habilidades sociales. Psicología Iberoamericana, 18(1), 6-18. https://doi. org/10.17979/reipe.2017.0.13.2131

IAB Spain (2019). Estudio anual de redes sociales 2019. https://tinyurl.com/vx5w8uc

Instituto Nacional de Estadística (2019). Población que usa internet. https://tinyurl.com/ tsplhs2

Lareki, A., Martínez de Morentín, J. I., Altuna, J., \& Amenábar, N. (2017). Teenagers' perception of risk behaviors regarding digital technologies. Computers in Human Behavior, 68, 395-402. https://doi.org/10.1016/j.chb.2016.12.004

Li, W., Garland, E. L., \& Howard, M. O. (2014). Family factors in Internet addiction among Chinese youth: A review of English- and Chinese-language studies, Computers in Human Behavior, 31, 393-411. https://doi.org/10.1016/j.chb.2013.11.004

Malo-Cerrato, S., Martín-Perpiñá, M., \& Viñas-Poch, F. (2018). Excessive use of social networks: Psychosocial profile of Spanish adolescents. [Uso excesivo de redes sociales: Perfil psicosocial de adolescentes españoles]. Comunicar, 56, 101-110. https://doi. org/10.3916/C56-2018-10

Mateo, J. (2012). La investigación ex post-facto. En R. Bisquerra (Coord.), Metodología de investigación educativa (195-229). La Muralla. https://tinyurl.com/suwm8p5

Mazzoni, E., Baiocco, L., Cannata, D., \& Dimas, I. (2016). Is internet the cherry on top or a crutch? Offline social support as moderator of the outcomes of online social support on Problematic Internet Use, Computers in Human Behavior, 56, 369-374. https:// doi.org/10.1016/j.chb.2015.11.032

Menezes M., Arvanitidis P., Smaniotto Costa C., \& Weinstein Z. (2019) Teenagers' Perception of Public Spaces and Their Practices in ICTs Uses. In: Smaniotto Costa C. et al. (eds) CyberParks - The Interface Between People, Places and Technology. vol 11380. Springer, Cham.

Merino Malillos, L. (2011). Jóvenes en redes sociales: significados y prácticas de una 
sociabilidad digital. Revista de Estudios de Juventud (95), 31-43. https://tinyurl.com/ ukta4ts

Morduchowicz, R. (2012). Los adolescentes y las redes sociales: la construcción de la identidad juvenil en Internet, Fondo de Cultura Económica.

Müller, K. W., Dreier, M., Beutel, M. E., Duven, E., Giralt, S., y Wölfing, K. (2016). A hidden type of internet addiction? Intense and addictive use of social networking sites in adolescents, Computers in Human Behavior, 55, 172-177. https://doi. org/10.1016/j.chb.2015.09.007

Pink, S., Horst, H., Postill, J., Hjorth, L., Lewis, T., \& Tacchi, J. (2016). Digital Etnography. Principles and practice. Sage.

Przybylski, A., Murayama, K., De Haan, C. R., \& Gladwell, V. (2013), Motivational, emotional, and behavioral correlates of fear of missing out, Computers in Human Behavior, 29(4), 1841-1848. https://doi.org/10.1016/j.chb.2013.02.014

Reig, D. (2012). Socionomía. ¿Vas a perderte la revolución social?, Ediciones Deusto.

Rojas-Jara, C., Henríquez, F., Sanhueza, F., Núñez, P., Inostroza, E., Solís, A., \& Contreras, D. (2018). Adicción a Internet y uso de redes sociales en adolescentes: una revisión. Revista española de drogodependencias, 43(4), 39-54. https://tinyurl.com/s9kdyb4

Scolari, C. (2013). Narrativas transmedia: cuando todos los medios cuentan, Deusto.

Rubio-Romero, J., Jiménez, J. M., \& Barón-Dulce, G. (2019). Las redes sociales digitales como espacios de sociabilidad de los adolescentes. El caso del colegio Escolapios de Aluche. Revista Mediterránea de Comunicación. 10(2), 85-99. https://www.doi. org/10.14198/MEDCOM2019.10.2.19

Tejada Garitan, E., Castaño Garrido, C., \& Romero Andonegui, A. (2019). Los hábitos de uso en las redes sociales de los preadolescentes. RIED. Revista Iberoamericana de Educación a Distancia, 22(2), 119-133 https://doi.org/10.5944/ried.22.2.23245

Tsitsika, A., Tzavela, E., \& Mavromatí, F. (2013). Research on Internet Addictive Behaviours among European Adolescents, Athens: EU NET ADB Project. https://tinyurl. com/wf7vthj

Vega-Almeida, R. L., \& Arencibia-Jorge, R. (2019). El lado oscuro de Internet: un estudio bibliométrico de la literatura sobre comportamientos adictivos en línea. Revista Española de Documentación Científica, 42(4), e251. https://doi.org/10.3989/ redc.2019.4.S8

Vernon, L., Barber, B. L., \& Modecki, K. L. (2015). Adolescent Problematic Social Networking and School Experiences: The Mediating Effects of Sleep Disruptions and Sleep Quality, Cyberpsychology, Behavior and Social Networking, 18(7), 386-392. https://doi.org/10.1089/cyber.2015.0107

Wang, J. L., Gaskin, J., Wang, H., \& Liu, D. (2016). Life satisfaction moderates the associations between motives and excessive social networking site usage, Addiction Research \& Theory, 24(6), 450-457. https://doi.org/10.3109/16066359.2016.1160283

We are social (enero, 2020). Digital 2020 España [Mensaje en un blog]. https://tinyurl. com/rgok6kd

Winocur, R. (2006). Internet en la vida cotidiana de los jóvenes. Revista Mexicana de Sociología, 68(3), 551-580. http://dx.doi.org/10.22201/iis.01882503p.2006.003.6069 
